\title{
Observations sur un Aselle obscuricole de France: Proasel/us racovitzai n.sp. (Crustacea /sopoda Asellota)
}

\author{
par \\ Jean-Paul HENRY et Guy MAGNIEZ*
}

\section{GENERALITES}

La grotte du Goueil-di-Her (Arbas, Haute-Garonne, Biospeologica no 1350), constitue un regard sur un important système hydrographique souterrain encore incomplètement exploré (Rouch, 1968, p. 71). La portion aval, seule visitable dans les conditions normales, se termine sur un vaste plan d'eau formant voûte mouillante et barrant l'accès au cours de la rivière souterraine située en amont (Lescher-Moutoué et Gourbault, 1970). Dès 1966, l'Asellote hypogé Stenasellus virei hussoni avait été observé sur le fond limoneux de la nappe d'eau (Magniez, 1968). En 1967, M.Cl.Bou captura un Asellide inconnu dans ce même siphon, ce qui incita l'un de nous (G.M.), accompagné de plusieurs membres du Laboratoire souterrain de Moulis, à retourner prospector la cavité. Le 17 août 1967, un lot très important de Sténaselles fût capturé et avec eux se trouvaient 11 Aselles de taille importante.

Ces Aselles étaient à peine pigmentés. Les yeux, très petits (2-3 éléments dissociés), existaient chez tous les individus. Un examen sommaire avait alors permis d'affirmer que l'espèce dont ils se rapprochaient le plus était la forme épigée pigmentée-oculée du Portugal septentrional: Proasellus ibericus (Braga, 1946). Depuis, pour la commodité, il nous était arrivé de désigner ces Aselles sous le nom de $P$. ibericus, en attendant de réaliser une étude approfondie de cette forme. (Magniez, 1969, 1970).

Or, grâce à la description récente de nouvelles espèces ( $P$. solanasi Henry et Magniez et $P$. coiffaiti Henry et Magniez en particulier), à la révision de l'ancien genre Asellus Geoffroy (Henry et Magniez, 1970) et à l'amélioration des techniques d'étude des pléopodes (Henry et Magniez, 1969), notre connaissance des Asellidae s'est grandement améliorée. Le système souterrain du Goueil-di-Her venant d'être l'objet d'une étude hydrologique et écologique extrêmement poussée (LescherMoutoué et Gourbault, 1970), un nombre important d'Aselles, en meilleur état de conservation, ont été capturés et nous ont été confiés par les deux auteurs que nous remercions très vivement de leur confiance. L'étude morphologique de la forme du Goueil-di-Her a pu être reprise en détail. Au même titre que les Asellides de la grotte de Sare (Proasellus coiffaiti Henry et Magniez), nous considérons ceux du Goueil-di-Her comme une espèce indépendante endémique. Malgré les nombreux

* Laboratoire de Biologie Animale et Générale, Université de Dijon, 6, Bd. Gabriel, 21 Dijon, France. 
travaux fondamentaux qu'il avait effectués sur les Asellidae et en particulier sur les formes françaises, aucune espèce de l'ancien Monde n'avait été dédiée jusqu'à présen à E.G. Racovitza. Nous réparons cet oubli en appelant Proasellus racovitzai n.sp. les Aselles du Goueil-di-Her, en rappelant que le grand zoologiste roumain avait très largement contribué à l'exploration des grottes de cette région nordpyrénéenne et même de la cavité dont il est question ici. Type de l'espèce: un ơ de $10,1 \mathrm{~mm}$, collections du Laboratoire souterrain de Moulis.

\section{DESCRIPTION SOMMAIRE DE PROASELLUS RACOVITZAI N.SP.}

Lots capturés: 8 ơ adultes de 6,6 à $10,1 \mathrm{~mm}$ et 9 ơ jeunes de 2,1 à $3,7 \mathrm{~mm}$; la différenciation des pléopodes sexuels semble s'effectuer pour des tailles comprises entre 2 et $3 \mathrm{~mm}$ environ. $7 q$ adultes à oostégites de repos génital, tailles de 5,1 à $7,5 \mathrm{~mm}$. 5 q à poche incubatrice vide de 6 à $7,5 \mathrm{~mm} .3$ ovigères de $5,8,6$ et $6,4 \mathrm{~mm}$. Celle de $6 \mathrm{~mm}$ (pl. photo $\mathrm{I} / 3$ ) portait 33 embryons sphériques de 290 à 310 microns de diamètre, taille comparable à celle de l'embryon au même stade de l'épigé $P$. meridianus. 7 jeunes $\$$ de 2 à $4,4 \mathrm{~mm}$; les oostégites sont encore invisibles dans cette catégorie de tailles. 47 très jeunes individus: ceux de $0,8 \mathrm{~mm}$ sont des larves, encore munies de leurs organes dorsaux et de leur cuticule embryonnaire, qui ont été prématurément expulsées du marsupium maternel; ceux de 1 à $1,4 \mathrm{~mm}$ sont encore démunis de péréiopodes VII; ceux de 1,5-1,6 $\mathrm{mm}$ portent des péréiopodes VII rudimentaires et ceux de 1,9-1,9 mm ont des péréiopodes VII parfaits.

Aspect général: Corps assez large et trapu, semblable à celui de l'espèce épigée $P$. meridianus ou à $P$. ibericus. Coefficient d'allongement de 3,5 environ, caractéristique des formes d'eau libre, alors que les interstitielles sont le plus souvent très longilignes (Pl. photo. I/4). Céphalon 1,5 fois plus large que long, avec une concavité rostrale marquée. Lobes postmandibulaires saillants et limite du somite

\section{PLANCHE I}

1) Proasellus racovitzai n.sp. $\$$ adulte de $6 \mathrm{~mm}$ du siphon de la grotte du Goueil di Her (Arbas, Haute-Garonne); (N. Gourbault et F. Lescher-Moutoué leg.) Remarquer l'absence quasi-totale de pigmentation sous-tégumentaire que certains individus conservent en partie. Les yeux, presque punctiformes et pigmentés intensèment sont présents. Les organes de Zenker sont très développes dans le péréion et le pléotelson. Un amas de cellules de Zenker particulier se développe dorsalement dans la tête et le premier péréionite libre. On le retrouve chez Proasellus meridianus; il pourrait être caractéristique des Proasellus de cette lignée.

2) Un autre individu (de $7 \mathrm{~mm}$ environ) de la même espèce dans lequel les amas de cellules de Zenker se développent davantage, en particulier dans la capsule céphalique. Chez les espèces épigées à pigmentation tégumentaire intense, ces formations sont en grande partie masquées.

3) Une o ovigère de $6 \mathrm{~mm}$ de la même espèce, en vue ventrale. Les péréiopodes III à VII ont été retirés pour faciliter l'observation de la poche incubatrice.

4) Proasellus albigensis (Magniez, 1965). đ̛ adulte de 4,5 $\mathrm{mm}$ de la source de la Mouline du Gô (Albi, Tarn); (Cl. Bou leg.). Proasellus racovitzai présente une forme typique d'Asellide d'eau libre (corps assez large, comme pour les espèces épigées). On voit qu'une espèce purement interstitielle est beaucoup plus grêle et allongée. Noter en particulier les différences d'allongement du pléotelson et des uropodes. 

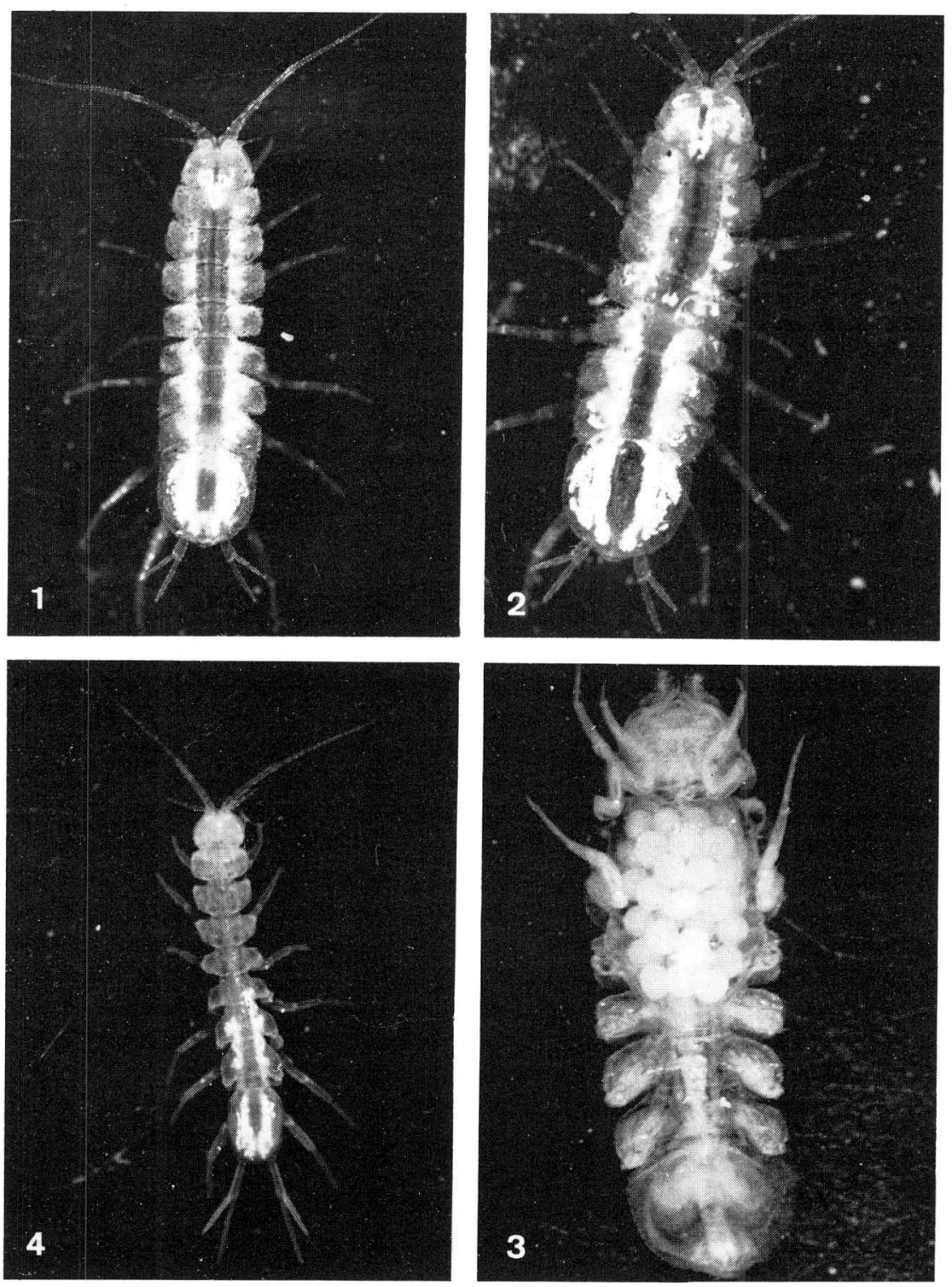
des maxillipèdes visible. Chez le ơ, largeur maximale du corps au niveau des péréionites V et VI $(2,5 \mathrm{~mm}$, alors qu'elle n'est que de 2,35 pour le VII et de 2,05 pour le pléotelson pour un o de $8,5 \mathrm{~mm}$ ). Coxopodites visibles en vue tergale, débordant latéralement les péréionites qui sont échancrés. Pléonites libres I et II étroits, en partie cachés par la marge caudale du péréionite VII. Pléotelson subcordiforme, légèrement décroissant caudalement, aussi large que long, mais un peu plus étroit que le péréionite VII. Pointe caudale bien marquée.

La plupart des individus, examinés à l'oeil nu, pourraient passer pour totalement dépigmentés, car ils sont apparemment blancs et tous les organes de Zenker sont parfaitement visibles par transparence. Toutefois, à la loupe binoculaire, un réseau très ténu de pigment sous-tégumentaire dorsal brun reste décelable. Quelques individus, surtout des $\delta$, capturés près des résurgences sont d'ailleurs notablement plus pigmentés. A l'oeil nu, ils semblent grisâtres et sont donc très semblables, du point de vue de la pigmentation aux Proasellus meridianus provenant de stations obscures (rivière souterraine de Padirac, sous-écoulement de la Dordogne, par exemple). $P$. racovitzai est donc une forme en 'état instable de dépigmentation' (Vandel, 1964, p.482), comparable aux populations d'Asellus aquaticus cavernicolus de la Piuka souterraine (Slovénie). Il serait intéressant de disposer d'individus vivants en nombre suffisant pour réaliser des élevages en milieu éclairé afin d'en étudier l'évolution chromatique.

Les yeux, punctiformes et pigmentés en brun, sont visibles sur tous les individus (cf. photographies 1 et 2 de la pl. I). Ils ne font pas saillie, si bien que l'exuvie antérieure de l'espèce pourrait passer pour celle d'une forme anophtalme. Le plus souvent, un ou deux amas pigmentaires et deux cornéules très réduites sont présents, ce qui pourrait correspondre à un état de régression oculaire assez semblable au stade $c$ défini par de Lattin (1939, fig. 2, p. 17) sur Asellus aquaticus cavernicolus. On trouve ici un nouvel exemple confirmant la remarque de Vandel (1964, p. 497): ' ... chez les cavernicoles, la disparition de la couleur du corps précède régulièrement celle des pigments oculaires...'. Il serait intéressant de pouvoir étudier également la sensibilité et les réactions de $P$. racovitzai à la lumière.

Les organes de Zenker sont très développés, surtout chez les adultes. Ils forment des amas blanc-jaunâtres dans tous les segments thoraciques ainsi que dans le pléotelson. Chez quelques individus de grande taille, ils paraissent s'étendre plus ou moins anarchiquement et envahir la cavité générale. On remarque en particulier la présence d'un amas impair fermé occupant la partie médio-postérieure de la tête et la partie médio-antérieure du péréionite I (photo.I/1). Cet amas semble caracté-

\section{PLANCHE 1.}

A - Extrémité d'une antennule d'un ơ de $9 \mathrm{~mm}$; e $=100$ microns.

$\mathrm{B}$ - Oostégite du maxillipède gauche d'une $९$ ovigère de $7,4 \mathrm{~mm} ; \mathrm{e}=200$ microns.

C - Péréiopode I d'un ơ de $8,7 \mathrm{~mm}$; e $=400$ microns.

D - La même, d'une 9 de $6,7 \mathrm{~mm} ; \mathrm{e}=200$ microns.

$\mathrm{E}$ - Un péréiopode IV d'un ơ de $8,5 \mathrm{~mm}$, appendice conformé en crochet nuptial, e $=400$ microns.

F - Marge latérale droite, en vue dorsale, du péréionite IV du même, montrant le débordement du coxopodite; $\mathrm{e}=\mathbf{4 0 0}$ microns. 


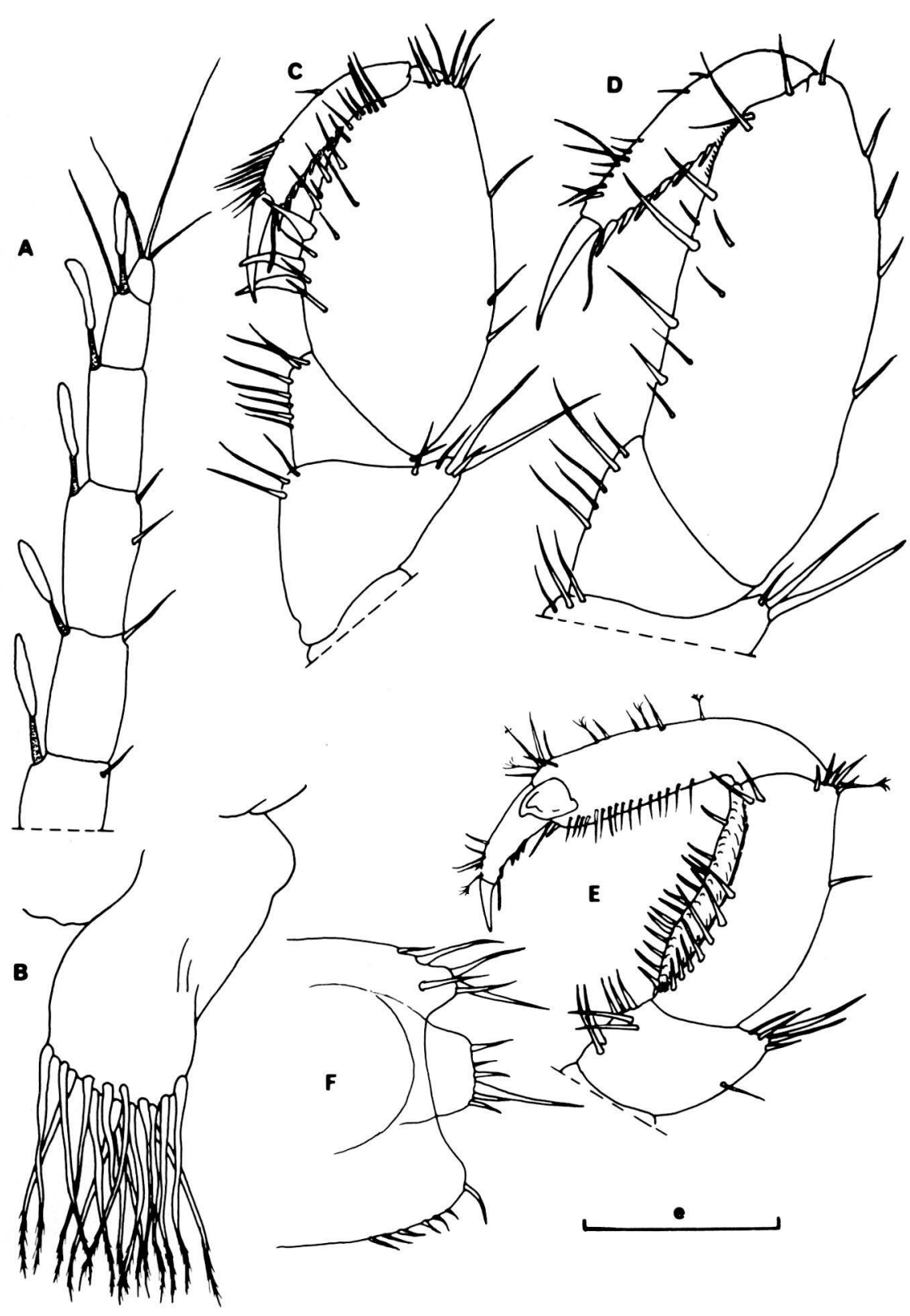


ristique de la lignée de $P$. meridianus, de même qu'un pont sternal entourant le rectum et joignant les organes de Zenker situés symétriquement dans les portions gauche et droite du pléotelson.

Appendices céphaliques: Caractéristiques du genre Proasellus. Antennules plus courtes que la hampe de l'antenne. Leur hampe compte 4 articles et leur fouet jusqu'à 15 articles chez les plus grands ot. Dans ce cas, les 5 distaux portent chacun une lame olfactive de 60 microns. Antennes atteignant $6,9 \mathrm{~mm}$ chez un of de $9 \mathrm{~mm}$. Hampe de 6 articles $(2,6 \mathrm{~mm})$ et fouet de 55 articles environ. Mandibules avec palpe triarticulé normales. Maxillules typiques avec lame interne à 5 tiges distales subégales. Maxilles normales.

Maxillipèdes:: Aspect caractéristique des Asellidae. Rétinacle avec 6 crochets de chaque côté (ठ) ou 4-5 (\$). Epipodite normal avec une quinzaine de courtes soies marginales lisses. Le maxillipède de la $q$ ovigère porte un oostégite ('Wasserstrudelapparat') aussi développé que celui des espèces épigées voisines et muni d'un faisceau de 14 longues tiges plumeuses à direction caudale.

Péréiopodes: Cas des mâles: les péréiopodes sont longs. Leur taille croît progressivement du I au VII, sauf pour le IV qui est très raccourci et différencié en crochet nuptial. Leurs longueurs respectives pour un ot de $9 \mathrm{~mm}$ sont en millimètres: 2,97; 4,$12 ; 4,35 ; 2,75 ; 6,13 ; 7,04 ; 7,32$ et leurs longueurs relatives en prenant le péréiopode II comme référence: $72,100,106,67,149,171$ et 178 . Le raccourcissement et la specialisation du péréiopode IV sont nettement moins marqués chez les ơ dont la taille est inférieure à $7 \mathrm{~mm}$. C'est donc seulement à partir de cette taille qu'ils acquéreraient leur aspect adulte définitif. Péréiopode I à propodite massif portant 3 phanères ensiformes au bord sternal. Dactylopodite avec une série continue de 8 épines sternales assez courtes. Les dactylopodites des péréiopodes II à VII portent généralement 4 épines sternales réparties en un groupe proximal de 3 épines plus une distale isolée. Chez les plus grands ot disponibles ( $9-10 \mathrm{~mm})$, les dactylopodites V, VI et VII possèdent une épine supplémentaire à la série proximale $(4+1)$. Cas des femelles: Longueurs respectives des péréiopodes d'une $q$ adulte de $6,7 \mathrm{~mm}: 2,00 ; 2,72 ; 2,80 ; 3,58 ; 4,10$ et $4,38 \mathrm{~mm}$. Longueur relative des mêmes: $74,100,103,109,132,147,161$. Péréiopode I sans phanères ensiformes au propodite et dactylopodite à 6 épines sternales. Dactylopodites des péréiopodes II à VII avec 2-3 épines sternales.

\section{PLANCHE 2:}

$\mathrm{G}$ - Pléopode I gauche, face sternale, d'un o de $9 \mathrm{~mm} ;=400$ microns.

$\mathrm{H}$ - Pléopode II gauche, face sternale, du même; $\mathrm{e}=200$ microns.

I - Extrémité de l'endopodite du pléopode II droit, d'un ơ de $8,5 \mathrm{~mm} ; \mathrm{e}=100$ microns.

$\mathrm{J}$ - Pléopode II gauche, face sternale, d'une $\$$ de $7,4 \mathrm{~mm} ; \mathrm{e}=400$ microns.

$\mathrm{K}$ - Pléopode III gauche d'un o de $9 \mathrm{~mm}$; $=1 \mathrm{~mm}$.

$\mathrm{L}$ - Pléopode IV gauche, face sternale, du même; $\mathrm{a}=$ linea area, $\mathrm{t}=$ linea transversalis; $\mathrm{e}=666$ microns.

$\mathrm{M}$ - Pléopode $\mathrm{V}$ gauche, face sternale, du même; $\mathrm{a}=$ linea articularis, $\mathrm{D}=$ linea duplex $; \mathrm{e}=666$ microns. 


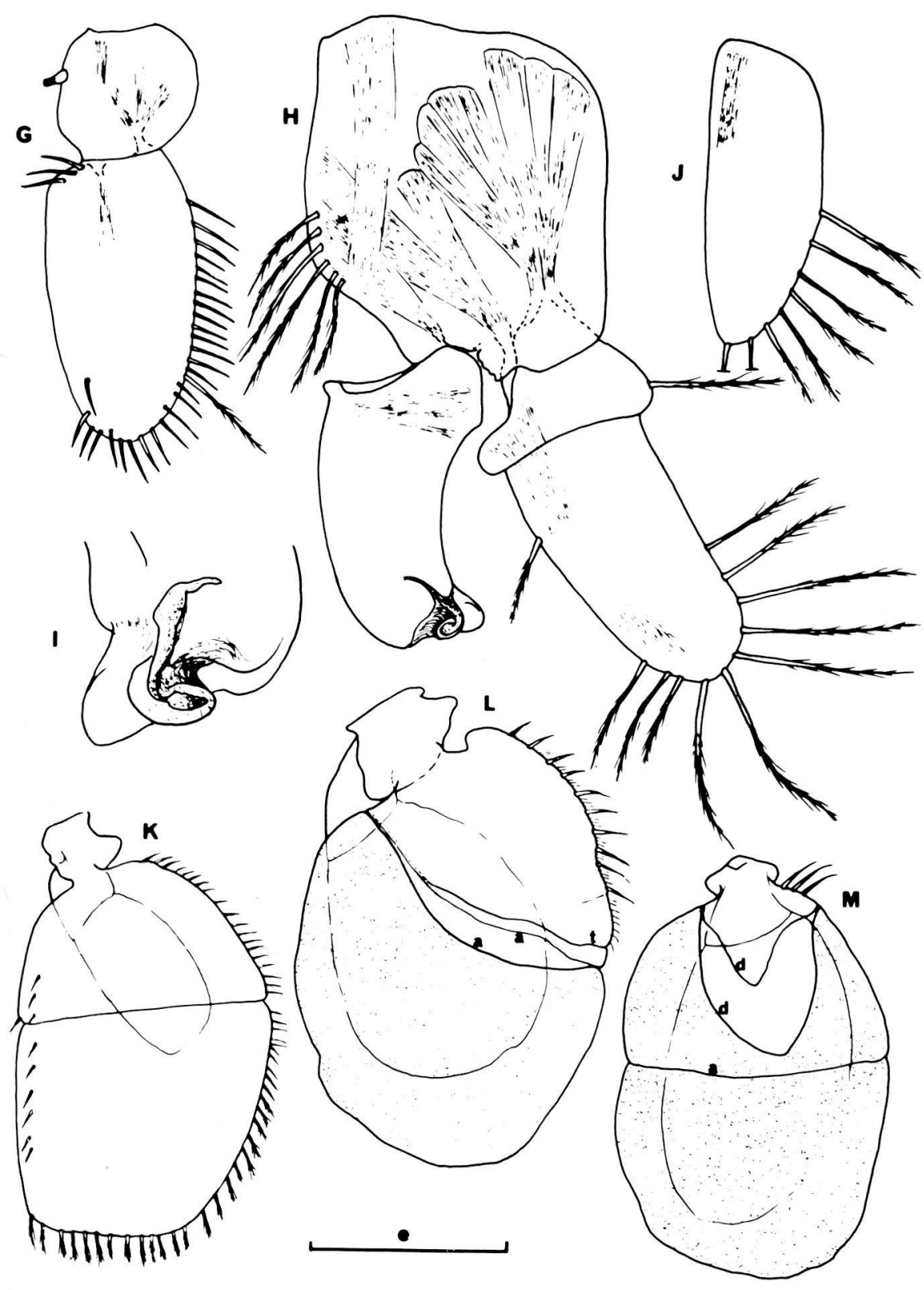


Pléopodes I o: Protopodite subtrapézoïdal, aussi long que large. Bord externe très convexe. Bord interne subrectiligne avec 1 , rarement 2 crochets rétinaculaires, à droite comme à gauche. Exopodite 2 fois plus long que large, portant 2-3 tiges lisses proximales internes, face sternale. Bords distal et interne garnis d'une série continue de 24-30 soies courtes, lisses et subégales. Dans la portion médiane de cette armature se trouvent en général 1-2 longues tiges plumeuses, rappel d'un stade ancestral. Enfin, 2 courtes soies lisses sternales sont implantées sur la portion distale de la rame.

Pléopodes II ठ: Protopodite subquadrangulaire, à peine plus long que large. Son bord interne, très convexe, porte 6 tiges plumeuses. Exopodite biarticulé, nettement plus long que l'endopodite. Article proximal court, à bord externe muni d'une seule soie plumeuse, rarement deux. Article distal 2 fois plus long que large, à bords subparallèles comme chez $P$. ibericus. 8-9 tiges plumeuses de 100 à 250 microns sur la marge distale et 2 tiges analogues sur la portion distale de la marge externe. La marge interne, normalement glabre chez la plupart des Proasellus, porte ici, en son milieu, une unique tige plumeuse, comme chez $P$. coiffaiti de la grotte de Sare. Une aire pilifère tergale existe, près du bord distal interne de l'article. Endopodite (organe copulateur) subcylindrique, aussi large, mais plus court que l'endopodite. Son aspect général rappelle celui de $P$. meridianus: portion proximale avec un fond légèrement excavé, sans apophyse interne; portion distale typique du genre et voisine de celle observée sur $P$. coiffaiti. Le goulot fendu, court et très large est nettement évasé distalement. Il montre un terme de passage entre le type $P$. ibericus ou $P$. coiffaiti et le type $P$. solanasi qui se rapproche davantage du terme de cette évolution ( $P$. meridianus). Apophyse tergale massive, à sommet arrondi et inclinée vers l'extérieur. L'angle entre les axes du goulot et de l'apophyse paraît voisin de $80^{\circ}$.

Pléopodes II ọ: Assez allongés, de forme subtriangulaire typique du genre. Leur bord externe porte une série continue de 8 longues tiges plumeuses.

Pléopodes III des deux sexes: Exopodite induré plus long que large, divisé en 2 articles par une suture à peine oblique. Article proximal plus petit que le distal. Marge externe et distale de la rame avec une rangée continue de tiges courtes. Celles de la moitié proximale sont lisses, les autres sont ciliées. Une rangée de soies submarginales internes sur la face sternale. Endopodite petit, subovalaire et charnu.

Pléopodes IV des deux sexes: Exopodite ovalaire presque 1 fois $1 / 2$ plus long que large, divisé en 2 articles. L'aire respiratoire (area) est très développée par suite de la migration de la linea area jusque contre la linea transversalis de la rame. Ces sutures, très rapprochées l'une de l'autre, restent encore bien individualisées sur la majeure partie de leur longueur, sauf vers le bord interne où elles se confondent. La linea conjungens, souvent invisible, est seulement amorcée vers le bord externe quand elle existe. Elle marque alors la limite d'une série de 10 tiges courtes et lisses, insérées sur la marge externe du premier article. Entre ces tiges, une bordure finement ciliée 
souligne la marge externe jusqu'à la limite de la linea transversalis. Endopodite charnu subcirculaire, plus petit que l'exopodite.

Pléopodes $V$ des deux sexes: Exopodite subcirculaire, $1 \frac{112}{2}$ fois plus long que large. La linea articularis et la linea duplex sont encore nettes et le divisent en 3 articles. La première suture, rectiligne, délimite un article distal charnu de surface importante. Par contre la linea duplex est très convexe, surtout sur la face sternale, ce qui réduit la hauteur de l'article intermédiaire, charnu, dans sa partie médiane. Le bord externe de l'article proximal, induré, porte 3 tiges lisses. Endopodite charnu ovalaire, moins long et moins large que l'exopodite.

Uropodes: Bien développés, ils sont à peine plus courts que le pléotelson chez le ỡ. Ceux de la $\uparrow$, proportionnellement, un peu moins longs. L'endopodite est légèrement plus long que l'exopodite, comme chez les autres espèces du genre.

\section{AFFINITES DE PROASELLUS RACOVITZAI}

Comme les deux espèces que nous avons décrites récemment ( $P$. solanasi, forme anophtalme d'Espagne méridionale et $P$. coiffaiti, forme oculée de la grotte de Sare), $P$. racovitzai s'inscrit naturellement dans la lignée évolutive du genre Proasellus qui a abouti à l'épanouissement de l'espèce moderne à vaste répartition: $P$. meridianus. On peut distinguer 6 stades:

1) A la base de cette lignée, il faut imaginer des formes dont l'organe copulateur aurait une structure assez semblable à ce que nous trouvons chez les 3 espèces endémiques suivantes:

- Proasellus ibericus (Braga, 1946), des eaux épigées du Portugal septentrional.

- P. infirmus (Birstein, 1936), forme oculée d'une source karstique de Transcaucasie occidentale.

- P. monsferratus (Braga, 1948), forme oculée d'une source de Kabylie. A ce stade, l'angle entre l'apophyse tergale et le goulot de l'organe copulateur est encore faible. Le diamètre du goulot reste médiocre et décroît distalement. Sa marge n'est pas encore réfléchie en corolle.

2) Le stade évolutif suivant de la lignée pourrait être représenté par les endémiques suivants:

- P. istrianus (Stammer, 1932), forme oculée des eaux souterraines de l'Istrie.

- P. remyi (Monod, 1932), forme littorale oculée du lac d'Ohrid.

- P. monodi (Strouhal, 1942), forme oculée d'une source karstique d'Epire. On note à ce stade l'existence d'un goulot court, mais de fort diamètre.

3) Chez les espèces suivantes:

- P. arnautovici (Remy, 1932), forme oculée littorale du lac d'Ohrid.

- P. coiffaiti Henry et Magniez 1972, forme oculée de la grotte de Sare.

- P. racovitzai n.sp., la marge distale du goulot a de plus en plus tendance à s'étaler perpendiculairement à l'axe longitudinal de l'organe. Le diamètre du goulot s'accroît donc distalement et l'aspect en corolle s'ébauche. 
4) - P. solanasi Henry et Magniez 1972, forme anophtalme de la province de Malaga nous montre un stade encore plus évolué. L'évasement du goulot s'accentue et l'angle entre les axes de celui-ci et de l'apophyse tergale s'accroît notablement.

5) - P. xavieri (Braga, 1956), forme oculée de l'étang de Fermentelos au Portugal, fournit un stade très évolué. La longueur et le diamètre du goulot se sont encore accrus. L'angle entre goulot et apophyse est devenu obtus. L'organe copulateur est preque identique à celui de l'espèce moderne $P$. meridianus qui semble marquer le terme de ce processus, (6ème stade).

Ce schéma a uniquement pour but de tracer les étapes probables de l'évolution qui a modelé le dispositif copulateur dans cette lignée d'Aselles. Pour P. racovitzai, il n'implique pas que l'espèce, sous sa forme actuelle, soit à l'origine de $P$. meridianus. En effet, $P$. racovitzai montre par ailleurs des caractères morphologiques de type cavernicole, comme l'extension des aires charnues sur les pléopodes IV et V. Ce n'est donc qu'un rameau latéral de l'arbre généalogique de $P$. meridianus. Il jalonne une étape intermédiaire de l'évolution du pléopode II. Cette étape a du être représentée, à une époque reculée, par des espèces épigées à vaste répartition.

La connaissance des Asellides et en particulier du genre périméditterranéen Proasellus n'a pu progresser que grâce à l'étude des espèces reliques pigmentées-oculées ou des espèces anophtalmes voisines. Les exemples récents de $P$. solanasi, $P$. coiffaiti et $P$. racovitzai montrent que l'inventaire faunistique est loin d'être clos, même dans des régions karstiques considérées comme bien explorées, car ces espèces sont endémiques, souvent cantonnées dans un réseau hydrographique restreint. Pour le Goueil-di-Her, il semble que $P$. racovitzai ne soit installé que dans les eaux libres de la zone de circulation permanente où les individus sont par ailleurs extrêmement disséminés (Lescher-Moutoué et Gourbault, 1970), alors que Stenasellus virei hussoni, qui cohabite avec l'Aselle, vit également dans les milieux interstitiels associés à cette zone, mais s'étend également vers le haut, dans la zone d'infiltration et de ruissellement souterrains du massif (Magniez, 1971). Les recherches entreprises sur les réseaux karstiques immédiatement voisins (Rouch, 1970), n'ont pas permis la mise en évidence de l'Aselle dans leurs eaux et nous ignorons les raisons qui s'opposent actuellement à l'extension de $P$. racovitzai dans le réseau de surface.

\section{PLANCHE 3:}

Schéma illustrant l'évolution probable de l'extrémité de l'endopodite des pléopodes II ơ dans la lignée du genre Proasellus menant à l'espèce moderne à vaste diffusion Proasellus meridianus (Rac.). La structure de cet organe copulateur chez certaines espèces anciennes, aujourd'hui endémiques, (Péninsule ibérique, Kabylie, lac d'Ohrid), correspond à plusieurs étapes de cette évolution. La lignée de $P$. meridianus inclut de plus de nombreuses espèces anophthalmes de la province ibéro-aquitaine, dont la différenciation a du être très précoce. Pour la clarté du schéma, leur endopodite n'a pas été figuré. 

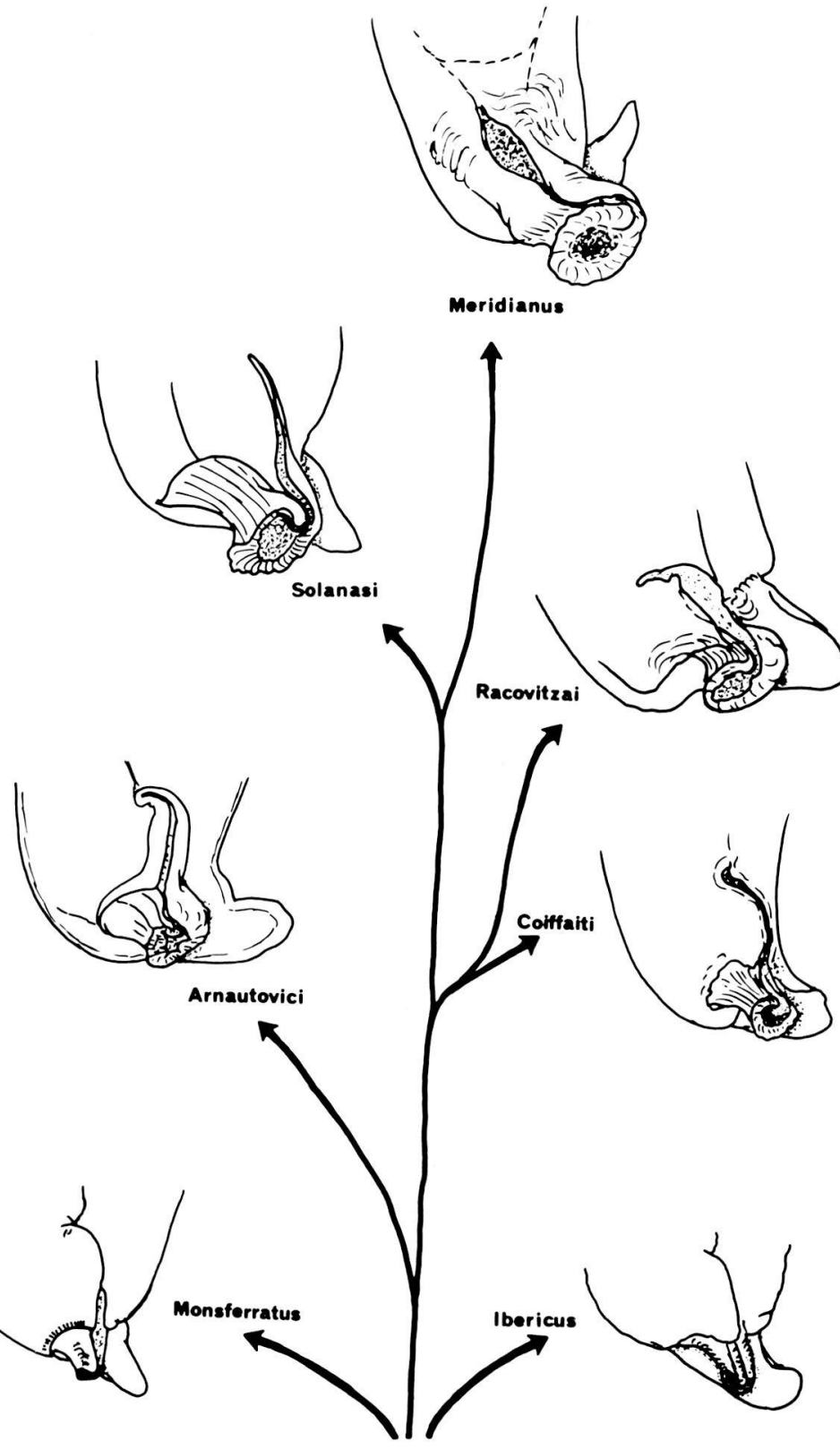


\section{DONNEES BIOLOGIQUES SOMMAIRES SUR LA NOUVELLE ESPECE}

Elles sont encore fragmentaires, car elles correspondent à des observations poursuivies durant quelques mois sur un très petit nombre d'individus. Il nous paraît cependant intéressant d'en faire mention, car elles permettent de mieux caractériser l'espèce.

L'élevage en laboratoire de cette espèce oculée souterraine est parfaitement réalisable. Les individus ont été maintenus à l'obscurité dans des chambres climatisées à $10^{\circ} \mathrm{C}$, soit environ $2^{\circ}$ au dessus de la température de leur biotope naturel. Ils sont nourris de feuilles d'Orme macérées ou de fragments de bois en décomposition, comme les espèces anophtalmes étudiées en même temps. $P$. racovitzai, comme les autres Aselles, est strictement végétarien. Dans les conditions d'élevage, la durée de l'intermue de l'Aselle adulte est du même ordre que celle des autres Asellides hypogés: environ 2 mois $1 / 2$ pour nos exemplaires. Ce ralentissement de son rythme de mue permet d'estimer que la longévité de l'espèce est de plusieurs années. A titre de comparaison, rappelons que les Aselles épigés, $P$. meridianus, par exemple, vivent généralement un an et que leur intermue dure une quinzaine de jours.

Quelques précisions sur la reproduction peuvent être apportées. Nous avons observé la formation de couples durables entre ot et $q$, ce qui semble indiquer que la fécondation est précédée d'une chevauchée nuptiale de durée indéterminée. Un tel comportement sexuel a été décrit chez les espèces de surface ( $A$. aquaticus, $P$. meridianus), mais non chez les Proasellus anophtalmes hypogés. Chez ces derniers, l'accouplement serait très bref et ne serait pas précédé de la formation de couples durables. Chez les Asellides cavernicoles, la même régression du comportement sexuel que chez les Amphipodes semble s'être opérée. En ce qui concerne $P$. racovitzai, l'étude morphologique du ơ laissait prévoir la subsistance d'une chevauchée nuptiale: les péréiopodes IV des grands $\delta$, transformés en crochets nuptiaux parfaits montrent que cette spécialisation anatomique est toujours fonctionelle.

Parmi les $\subsetneq$ mises en élevage début janvier 1971, nous avons obtenu une ponte, courant février de la même année. Le nombre d'oeufs contenus dans le marsupium de cette $q$ de $6 \mathrm{~mm}$ était de 12. Par contre, une autre $q$ récoltée ovigère $(6 \mathrm{~mm}$ également), portrait 33 embryons. Pour la $\subsetneq$ en élevage, la durée d'incubation est comprise entre 7 et 11 semaines, la date exacte de la ponte n'étant pas connue. Cette durée est très voisine de celles des Proasellus hypogés (Proasellus cavaticus, par exemple), relevées par l'un de nous (J-P.H.). Elle est au contraire nettement plus longue que les durées d'incubation des espèces épigées (15 jours environ, à température normale).

\section{PLANCHE II}

1) Chromosomes somatiques (plaque métaphasique) de Proasellus meridianus. Préparation réalisée à partir d'un oeuf en cours de segmentation. $\mathrm{e}=6,5 \mu$

2) Chromosomes somatiques (plaque métaphasique) de Proasellus racovitzai. Préparation réalisée à partir d'un oeuf en cours de segmentation. e $=10 \mu$

3) Pléopode II gauche, face sternale, d'un mâle de Proasellus racovitzai. e $=150 \mu$.

4) Endopodite (organe copulateur) de l'apprendice précédent. L'évasement distal du goulot, caractéristique de cette lignée de Proasellus, est bien visible. e $=53 \mu$. 

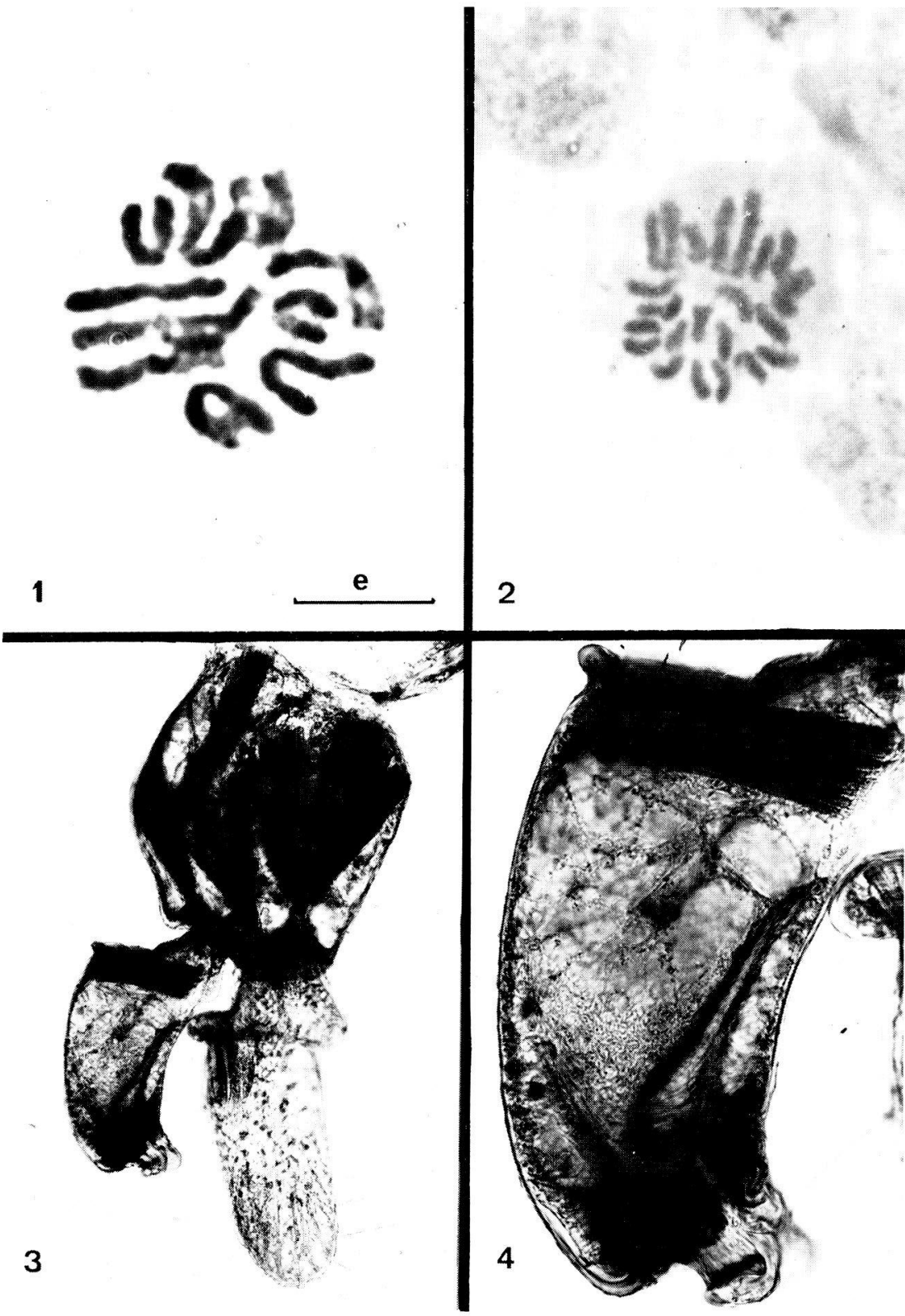
Comme chez tous les Asellides, les jeunes quittent la poche incubatrice à l'état larvaire, puis subissent plusieurs mues dans les semaines suivantes pour acquérir leur morphologie d'adulte. Les caractères de ces différents stades seront précisés prochainement dans le cadre d'une étude détaillée du développement postembryonnaire de $P$. cavaticus (J-P.H.). Ici, le rythme des mues et la croissance des jeunes rappellent ceux des espèces anophthalmes. 6 embryons de la $q$ étudiée ont été extraits du marsupium pour l'étude du lot chromosomique de l'espèce, les 6 autres sont nés presque simultanément. 6 jours après la libération, la $q$ a rejeté son exuvie postérieure. L'exuvie antérieure a suivi 48 heures après. Ces durées pour la mue postgénitale sont les mêmes que pour d'autres Proasellus souterrains, comme $P$. cavaticus. Au cours de cette mue, la $q$ perd sa poche incubatrice et il ne subsiste que des oostégites non fonctionnels, de taille réduite, dont l'aspect rappelle ceux des $q$ de $P$. meridianus au repos sexuel. Le maintien en élevage de cette $\$$ a permis d'avoir une idée du rythme des reproductions. Cet Aselle a subi une nouvelle mue parturielle en septembre 1971 et a pondu quelques oeufs. On pourra donc envisager l'existence de deux pontes par an dans l'espèce. Ces résultats demandent à être confirmés par des élevages plus durables sur un matériel plus abondant, mais ils laissent néanmoins prévoir un cycle biologique de type cavernicole pour $P$. racovitzai.

Equipement chromosomique de la nouvelle espèce: Les données caryologiques sur les Asellides restent fragmentaires. Elles concernent principalement les trois espèces épigées communes: Asellus aquaticus, Proasellus meridianus et $P$. coxalis, dont de nombreux auteurs ont déterminé les lots chromosomiques $(2 n=16$ pour le premier, $2 n=10$ pour le second et $2 n=12$ pour le troisième). On a eu tendance à penser que ces faibles nombres étaient valables pour l'ensemble des Asellides.

Une seule forme souterraine avait été étudiée jusqu'à présent ( $P$. cavaticus) dont l'un de nous (Henry, 1967) a donné sans ambiguïté le nombre chromosomique $(2 n=24)$. Dans les conditions actuelles, il est bien difficile de se faire une idée exacte de l'évolution des garnitures chromosomiques des Asellidae. Il faudrait pour cela connaître le nombre de chromosomes des espèces oculées endémiques citées plus haut, ainsi que celui des formes anophtalmes. Pour apporter une donnée supplémentaire dans ce domaine, nous avons étudié l'équipement chromosomique de $P$. racovitzai, forme dont nous avons par ailleurs souligné l'intérêt phylogénétique.

Le nombre de chromosomes somatiques a été établi par l'examen des premières divisions de segmentations de l'oeuf selon les techniques habituelles d'études des chromosomes. Le dénombrement a été assez facile, comme en témoigne la photographie no 2, Planche II représentant une plaque métaphasique où sont bien individualisés 22 éléments. L'équipement chromosomique de $P$. racovitzai s'établit donc à $2 \mathrm{n}=22$.

Un chiffre voisin $(2 n=24)$ s'observe chez $P$. cavaticus, alors que les espèces modernes de Proasellus épigés présentent des nombres beaucoup plus faibles (cf. Planche II/1). On peut donc penser que les formes primitives du genre Proasellus possédaient un nombre chromosomique relativement élevé $(2 n=24$ chez $P$. cavati- 
cus, par exemple). Ce nombre s'est considérablement réduit chez les espèces actuelles à vaste répartition. Malheureusement, les renseignements que nous possèdons sont encore trop succincts pour tenter d'établir l'évolution d'une formule à l'autre, en particulier pour faire dériver la formule de $P$. meridianus (10) de celle de $P$. racovitzai, dont l'étude morphologique nous a pourtant montré sans ambiguité l'étroite parenté.

\section{RAPPEL DE LA LISTE DES ESPECES D’ASELLIDES DE FRANCE}

La faune d'Asellidae de France est constituée principalement par des espèces (16), soit épigées, soit hypogées, du genre Proasellus Dudich. On y rencontre de plus la forme oculée très commune dans la moitié Nord du pays: Asellus (Asellus) aquaticus (L) sensu Racovitza, 1919, qui est très isolée dans la faune d'Europe occidentale, puisque la presque totalité des représentants du genre Asellus n.def. se trouvent dans l'Extrême-Orient boréal et au Japon; ainsi que l'espèce anophtalme endémique Conasellus (?) heilyi (Legrand, 1956), d'une rivière souterraine du Poitou, qui présente des affinités avec les genres nord-américains ou le genre lusitanien Bragasellus.

\section{Liste des Proasellus oculés de France}

1) Proasellus coxalis (Dollfus, 1892), subsp. banyulensis Racovitza, 1919: eaux épigées des fleuves côtiers méditerranéens, des Pyrénées-orientales aux Alpesmaritimes; souvent dans les eaux souterraines de la même région.

2) Proasellus meridianus (Racovitza, 1919): forme autochtone des eaux épigées de l'Ouest de la France. Des peuplements dissociés dans les bassins de la Loire (Vienne, Creuse, rivières du Morvan), de la Seine (Douix de Chatillon), de la Saône et du Rhin. Nombreuses populations dans les eaux souterraines (gouffre de Padirac, grotte de St-Géry, puits en Bretagne, etc ...). Semble absente dans l'aire de P. coxalis.

3) Proasellus coiffaiti Henry et Magniez, 1972: forme endémique des grottes de Sare (Pyrénées-Atlantiques).

4) Proasellus racovitzai n.sp: forme endémique du système karstique du Goueil-diHer (Haute-Garonne).

\section{Liste Des Proasellus Anophtalme de France}

\section{Groupe Cavaticus}

5) Proasellus cavaticus (Leydig, 1871), sensu Henry 1971: eaux souterraines du Nord-Est de la France, avec des stations karstiques plus méridionales.

6) Proasellus valdensis (Chappuis, 1948): eaux souterraines du Jura méridional et des Préalpes calcaires. 


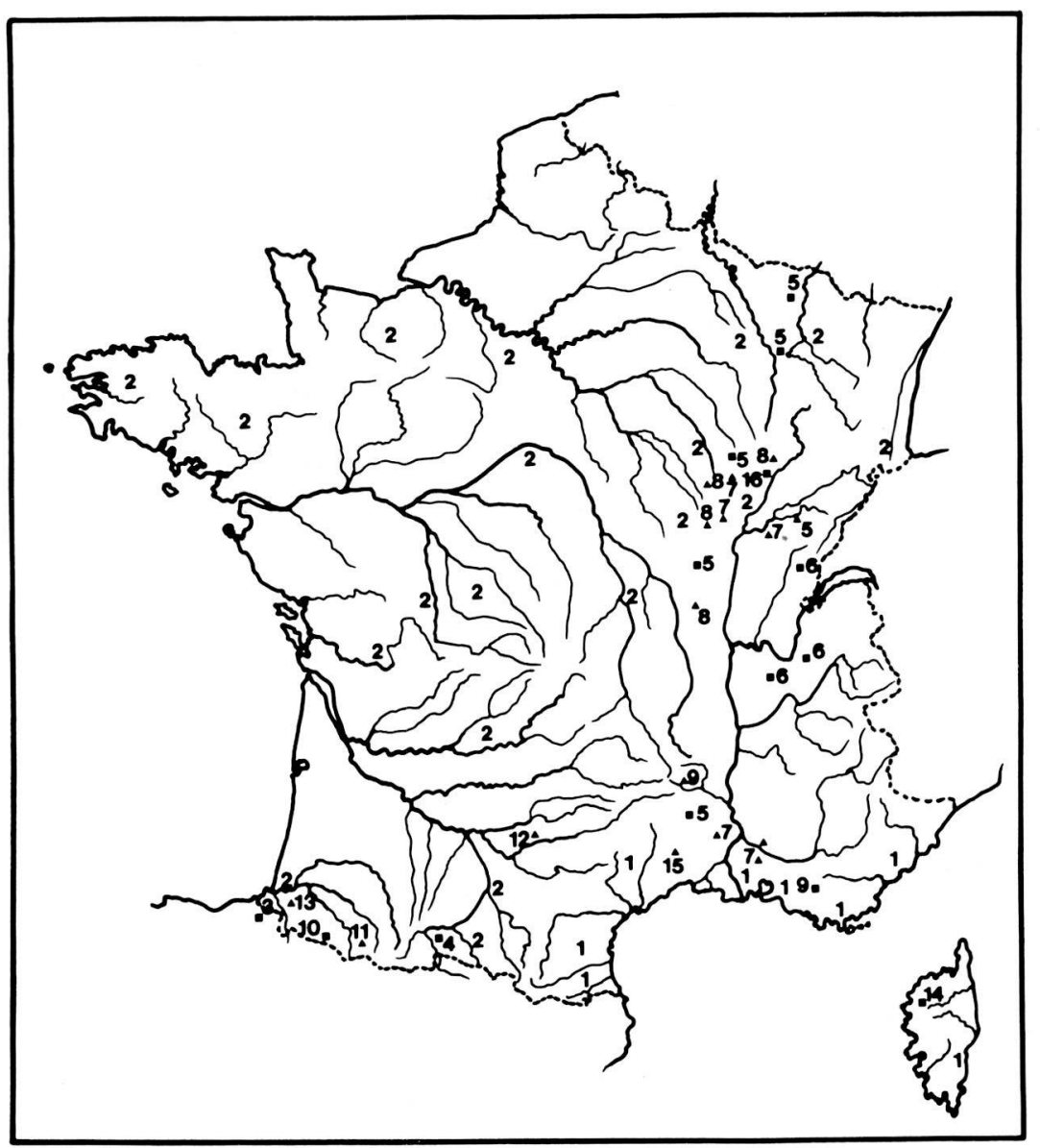

\section{PLANCHE 4:}

Carte schématique de la France, avec les principaux réseaux hydrographiques. Les différentes espèces du genre Proasellus sont notées avec le même numéro que dans la liste incluse dans la texte: $1=P$. coxalis, subsp. banyulensis ou coxalis; $2=P$. meridianus. Dans le cas de ces espèces épigées à vaste diffusion, la localisation des chiffres correspond en général à un peuplement régional généralisé et non à une simple station. $3=P$. coiffaiti $; 4=P$. racovitzai n.sp.; $5=P$. cavaticus; $6=\mathrm{P}$. valdensis; $7=P$. walteri; $8=P$. strouhali subsp. puteanus; $9=$ P. synaselloides; $10=P$. spelaeus; $11=P$. spelaeus aquae-calidae $; 12=P$. albigensis $; 13=P$. vandeli $; 14=P$. beroni $;$ $15,=P$. boui $; 16=P$. burgundus. Les stations des espèces souterraines sont indiquées par des figurés: carré noir = biotope karstique, triangle noir = biotope phréatique. 
7) Proasellus walteri (Chappuis, 1948): petite forme interstitielle des nappes alluviales des bassins de la Saône et du Rhône.

8) Proasellus strouhali (Karaman, 1955), subsp. puteanus Henry 1966: nappes phréatiques de Bourgogne et régions avoisinantes.

9) Proasellus synaselloides (Henry, 1963): eaux souterraines du Var et de l'Ardèche.

Groupe à affinités ibériques (lignée de P. meridianus)

10) Proasellus spelaeus (Racovitza, 1922): eaux souterraines du massif des Arbailles (Pyrénées-Atlantiques).

11) Proasellus spelaeus aquae-calidae (Racovitza, 1922): eaux souterraines des vallées du Gave d'Ossau et du Gave d'Aspe (Pyrénées-Atlantiques).

12) Proasellus albigensis (Magniez, 1965): forme allongée montrant une nette adaptation à la vie interstitielle. Nappes alluviales du Tarn.

13) Proasellus vandeli Magniez et Henry, 1969: eaux souterraines du bassin du Gave de Mauléon (Pyrénées-Atlantiques).

Formes à affinités balkaniques:

14) Proasellus beroni Henry et Magniez, 1968: eaux souterraines de Corse centrale.

15) Proasellus boui Henry et Magniez, 1969: nappes phréatiques du Gardon d'Alès (Gard).

16) Proasellus burgundus Henry et Magniez, 1970: rivière souterraine de Bèze (Côte-d'Or).

(Manuscrit terminé le 9 octobre 1971).

\section{RESUME}

Description d'une espèce nouvelle du genre Proasellus Dudich. Proasellus racovitzai n.sp. est très largement dépigmenté, possède des yeux réduits et mène une vie troglobie dans le système hydrographique souterrain du Goueil-di-Her (Haute-Garonne, France). L'espèce est un représentant endémique ancien de la lignée évolutive menant à la forme épigée moderne $P$. meridianus (Racovitza). Nombre chromosomique de la nouvelle espèce: $2 \mathrm{n}=22$.

\section{SUMMARY}

Description of a new species of the genus Proasellus Dudich. Proasellus racovitzai n.sp. is widely depigmented. The eyes are reduced. It lives in the underground stream of the Goueil-di-Her cave system (Haute-Garonne, France). The species is an old, endemic form of the phyletic line of the modern epigean species $P$. meridianus (Racovitza). Chromosome number of the new species: $2 n=22$. 


\section{BIBLIOGRAPHIE}

BRAGA, J.M. - 1946 - Quelques Asellides nouveaux du Portugal. Mem. e. Est. Mus. Zool. Univ. Coimbra, 173, 1-25.

HENRY, J.P., 1967 - Le nombre de chromosomes de l'Isopode hypogé Asellus cavaticus Leydig. C.R. Acad. Sc. Paris, 264, 2126-29.

HENRY, J.P., 1970 - Contribution à l'étude du genre Proasellus (Crustacea Isopoda Asellidae), le groupe cavaticus. Vie et Milieu, Paris, (sous presse).

HENRY, J.-P., 1970 - Données récentes sur la faune hypogée d'Asellides de France. Actes 96ème Congrès Nat. Soc. Sav. Toulouse, (sous presse).

HENRY, J.-P. et MAGNIEZ, G., 1969 - Etude au microscope électronique à balayage des pléopodes copulateurs de deux espèces de Proasellus (Crustacea Isopoda Asellota). C.R. Acad. Sc. Paris, 26S, 718-720.

HENRY, J.-P. et MAGNIEZ, G., 1970 - Contribution à la systématique des Asellides. Ann. Spéléol. Paris, 25, 2, 335-367.

HENRY, J.-P. et MAGNIEZ, G., 1972 - Un Aselle endémique pigmenté et oculé de France: Proasellus coiffaiti n.sp. (Crustacea Isopoda Asellota), Ann. Spéléol. Paris, (sous presse).

HENRY, J.-P. et MAGNIEZ, G., 1972 - Un Aselle cavernicole d'Espagne méridionale: Proasellus solanasi n. sp. (Crustacea Isopoda Asellota). Int. J. Speleol. 4, 1 : 37-44.

HYNES, H.B.N., 1959 - On the occurrence of Gammarus duebeni Lilljeborg in freshwater and of Asellus meridianus Racovitza in western France. Hydrobiologia, XIII, 1/2, 152-155 .

LESCHER-MOUTOUE, F, et GOURBAULT, N., 1970 - Etude écologique du peuplement des eaux souterraines de la zone de circulation permanente d'un massif karstique. Ann. Spéléol. Paris, 25, 4, 765-848.

MAGNIEZ, G., 1967 - 1968 - Les stations de Stenasellus virei Dollfus (Crustacé Isopode troglobie). Sous le Plancher, Dijon, VII/1,1-11.

MAGNIEZ, G., 1969 - Données récentes sur quelques Asellotes hypogés (Crustacea Isopoda). Spelunca, Paris, Mem. no. 6, p. 87.

MAGNIEZ, G., 1970 - Sur un Aselle cavernicole de France: Proasellus ibericus (Braga, 1946). Sous le Plancher, Dijon, IX/2,21-25.

MAGNIEZ, G., 1971 - Les stations de Stenasellus virei Dollfus (Crustacé Isopode troglobie). Sous le Plancher, Dijon, X/1, 13-25.

MONOD, Th., 1932 - Sur un Asellus aberrant (A. remyi nov. sp.) du lac d'Ohrid (Albanie). Bull. Soc. Zool. France, 57, 3, 206-217.

ROUCH, R., 1968 - Contribution à la connaissance des Harpacticides hypogés (Crustacés Copépodes). Ann. Spéléol. Paris, 23, 1,5-167.

ROUCH, R., 1971 - Peuplement par les Harpacticides d'un drain situé dans la zone de circulation permanente. Ann. Spéléol. Paris, 26, 1, 107-133.

VANDEL, A., 1964 - Biospéologie - La biologie des animaux cavernicoles. Gauthier-Villars, Paris, 1-619. 